\title{
Point groups in solid state physics I: point group $O_{h}$
}

\author{
Ayse Delibas, Vildan Aykan, Deniz Turkkan, Harun Akkus* \\ Physics Department, Faculty of Science, Yuzuncu Yil University, 65080 Van, Turkey
}

Email address:

physicisthakkus@gmail.com(H. Akkus)

\section{To cite this article:}

Ayse Delibas, Vildan Aykan, Deniz Turkkan, Harun Akkus. Point Groups in Solid State Physics I: Point Group $\mathrm{O}_{\mathrm{h}}$, American Journal of Modern Physics. Vol. 2, No. 2, 2013, pp. 81-87. doi: 10.11648/j.ajmp.20130202.19

\begin{abstract}
The definitions of all symmetry operations, their permutation counterparts and matrix representations, the multiplication table, all classes, abelian subgroups, and some other subgroups of point group $\mathrm{O}_{\mathrm{h}}$ are clearly obtained. For every symmetry operation (or element) of $\mathrm{O}_{\mathrm{h}}$, appropriate figures are pictured for the sake of clarity.
\end{abstract}

Keywords: Point Groups, Solid State Physics, Symmetry

\section{Introduction}

Group theory studies the groups in mathematics. A group in mathematics is both an algebraic and an abstract structure. As an abstract structure a group is a set of elements. The set of elements must satisfy the following four axioms, which are called "group axioms", with a law of composition or multiplication to be a group. (1) Closure: the product of any two elements of the set with the law of composition, must be one of the elements in the set, that is, the result of the multiplication is itself an element which has to appear in the set. (2) Unit element: the set must include a unit element such that the product of any element in the set with the unit element according to the law of composition of set leaves the element unchanged, for example, for any element a, should be $\mathrm{ae}=\mathrm{ea}=\mathrm{a}$ where $\mathrm{e}$ is the unit element of the set. (3) Inverse element: a inverse element must exist for every element in the set and the set has to include all inverse elements, for example, for any element $\mathrm{a}, a a^{-1}=$ $a^{-1} a=e$ where $a^{-1}$ is the inverse element of a. (4) Associative law: the set must exhibit the associative law, for example, for any three elements $\mathrm{a}, \mathrm{b}$, and $\mathrm{c}, \mathrm{a}(\mathrm{bc})=(\mathrm{ab}) \mathrm{c}$ has to be provided. Furthermore, if all elements in the group commute, that is, for any two elements $a b=b a$, then the group is called an Abelian group.

There exist a lot of applications of the group theory in physics. One of important application area is solid state physics. The crystals in solid state physics are divided into thirty two classes according to their point groups or symmetry groups. Although the point groups are often used in the solid state physics and involved in many solid state physics textbooks [1-15], realizing them is hard because the imagination of successive applications of the symmetry operations is complicated. The aim of this paper is to provide a better understanding of the point groups used in solid state physics. But we have restricted the paper to the cubic point group $\mathrm{O}_{\mathrm{h}}$. We have worked the other cubic point groups $\mathrm{O}, \mathrm{T}, \mathrm{T}_{\mathrm{h}}$, and $\mathrm{T}_{\mathrm{d}}$ as well, however, we have made them the topics of another paper unpublished yet.

\section{Symmetry elements of $O_{h}$}

In crystal physics, a symmetry element is a symmetry operation that converts the crystal structure into itself. A symmetry group is a group which consists of symmetry operations. A point group is also a symmetry group but its elements leave one point of the crystal unchanged.

Symmetry group $\mathrm{O}_{\mathrm{h}}$ and one of the subgroups of permutation group $\mathrm{P}(8)$ are isomorphic groups. We can learn and investigate the point group $\mathrm{O}_{\mathrm{h}}$ using the isomorphism between these groups. Using the isomorphism between $\mathrm{O}_{\mathrm{h}}$ and the subgroup of $\mathrm{P}(8)$ enables us to define every symmetry element of $\mathrm{O}_{\mathrm{h}}$. To do this, first let us point the corners of a cube with the digits from one to eight. Fig. 1 shows such a cube. Three twofold axes $\left(\mathrm{C}_{2}\right)$, eight threefold axes $\left(C_{3}\right)$, and six fourfold axes $\left(C_{4}\right)$ are also shown in this figure. To avoid confusion, we mark the symmetry axes, $\mathrm{C}_{2}$, $\mathrm{C}_{3}, \mathrm{C}_{4}$, with the digits parenthetically in Fig. 1. Now, let us define the symmetry elements which leave the cube unchanged. 


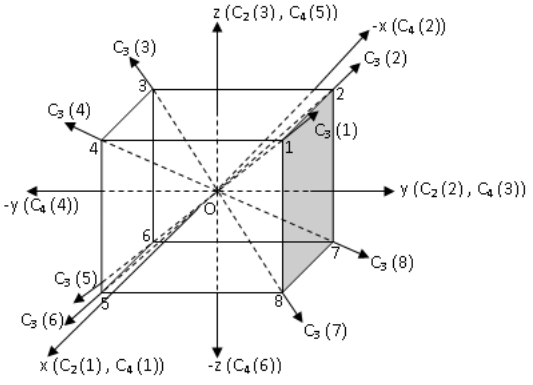

Figure 1. Illustration of two-fold $\left(C_{2}\right)$, three-fold $\left(C_{3}\right)$, and four-fold $\left(C_{4}\right)$ axes for a cube.

We match every symmetry operation (or symmetry element) in the group Oh with an element in $\mathrm{P}(8)$. The first symmetry element is naturally identity element, e in Table 1 and its counterpart is the element of $\left(\begin{array}{l}12345678 \\ 12345678\end{array}\right)$ in $P(8)$. The second symmetry element a in Table 1, is a proper rotation through $120^{\circ}$ about $\mathrm{C} 3$ (1) axis in Fig. 1 and its counterpart is element of $\left(\begin{array}{l}12345678 \\ 14587632\end{array}\right)$ in the group P (8). However, we can use briefer symbols for simplicity. That is why we can use symbols (12345678) and (14587632) to denote the elements e and a, respectively. For a better understanding the symmetry operations which will be defined, for example, we have demonstrated the effect of the operation a on a cube in Fig. 2. The symbols of counterparts in $P$ (8) and definitions of all symmetry elements which are the proper rotations through $120^{\circ}$ about $\mathrm{C} 3$ axes in Fig. 1 are given in Table 1 . They are elements $a, b, c, d, f, g, h$, and $i$ in Table 1. The symbols $\mathrm{j}, \mathrm{k}$, and 1 in Table 1 represent the elements which are the proper rotations through $180^{\circ}$ about C2 (1) (or x), C2 (2) (or y), and C2 (3) (or z) axes in Fig. 1, respectively. As to the elements $\mathrm{m}, \mathrm{n}, \mathrm{o}, \mathrm{p}, \mathrm{r}$, and $\mathrm{s}$ in Table 1 , they are the proper rotations through $90^{\circ}$ about $\mathrm{C} 4$ (1) (or x), C4 (2) (or -x), C4 (3) (or y), C4 (4) (or -y), C4 (5) (or z), and C4 (6) (or -z) axes in Fig. 1, respectively. Fig. 3 shows different six symmetry axes $C_{2}^{\prime}(1,2, \ldots, 6)$. The symmetry elements which are the proper rotations through $180^{\circ}$ about these axes are represented symbols t, u, v, w, y, and $\mathrm{z}$ in Table 1.

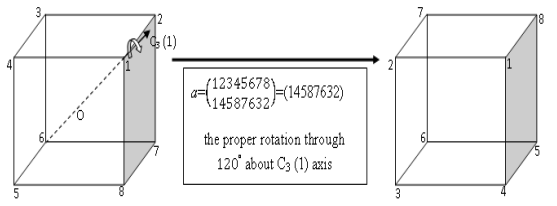

Figure 2. Illustration of symmetry element a.

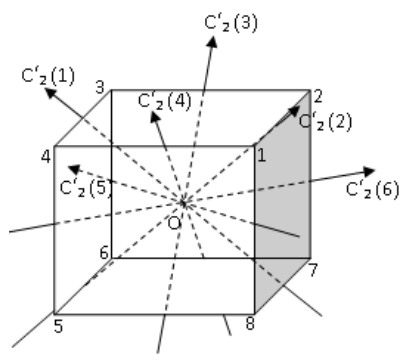

Figure 3. Illustration of two-fold $C_{2}^{\prime}$ axes.

Table 1. Symmetry elements of point group $O_{h}$ and their permutation counterparts and matrix representations.

\begin{tabular}{|c|c|c|c|c|c|c|}
\hline Symbol & $\begin{array}{l}\text { Permutation } \\
\text { counterpart }\end{array}$ & $\begin{array}{l}\text { Matrix represen } \\
\text { tion }\end{array}$ & Definition & $\begin{array}{l}\text { Permutation } \\
\text { counterpart }\end{array}$ & $\begin{array}{l}\text { Matrix representa- } \\
\text { tion }\end{array}$ & Definition \\
\hline e & (12345678) & $\left(\begin{array}{lll}1 & 0 & 0 \\
0 & 1 & 0 \\
n & n & 1\end{array}\right)$ & Identity element & (65872143) & $\left(\begin{array}{ccc}-1 & 0 & 0 \\
0 & -1 & 0 \\
n & n & 1\end{array}\right)$ & $\begin{array}{l}\text { Inversion through the origin } \\
\text { (point } \mathrm{O} \text { or centre of the cube). }\end{array}$ \\
\hline $\mathrm{a}$ & (14587632) & $\left(\begin{array}{lll}0 & 0 & 1 \\
1 & 0 & 0 \\
0 & 1 & 0\end{array}\right)$ & $\begin{array}{l}\text { Proper rotation through } \\
120^{\circ} \text { about } C_{3} \text { (1) axisa } \\
\text { (fig. } 1 \text { ). }\end{array}$ & (67234185) & $\left(\begin{array}{ccc}0 & 0 & -1 \\
-1 & 0 & 0 \\
0 & -1 & 0\end{array}\right)$ & $\begin{array}{l}\text { Inversion after the symmetry } \\
\text { element (or operation) a (fig. } 1 \text { ). }\end{array}$ \\
\hline b & (72185436) & $\left(\begin{array}{ccc}0 & -1 & 0 \\
0 & 0 & 1 \\
-1 & 0 & 0\end{array}\right)$ & $\begin{array}{l}\text { Proper rotation through } \\
120^{\circ} \text { about } C_{3}(2) \text { axisb }^{*} \\
\text { (fig.1). }\end{array}$ & (45632781) & $\left(\begin{array}{ccc}0 & 1 & 0 \\
0 & 0 & -1 \\
1 & 0 & 0\end{array}\right)$ & $\begin{array}{l}\text { Inversion after the symmetry } \\
\text { element (or operation) b (fig.1). }\end{array}$ \\
\hline $\mathrm{c}$ & (76321458) & $\left(\begin{array}{ccc}0 & 0 & -1 \\
1 & 0 & 0 \\
0 & -1 & 0\end{array}\right)$ & $\begin{array}{l}\text { Proper rotation through } \\
120^{\circ} \text { about } C_{3} \text { (3) } \text { axisc }^{*} \\
\text { (fig.1). }\end{array}$ & (41856723) & $\left(\begin{array}{ccc}0 & 0 & 1 \\
-1 & 0 & 0 \\
0 & 1 & 0\end{array}\right)$ & $\begin{array}{l}\text { Inversion after the symmetry } \\
\text { element (or operation) c (fig.1). }\end{array}$ \\
\hline d & (36541872) & $\left(\begin{array}{ccc}0 & -1 & 0 \\
0 & 0 & -1 \\
1 & 0 & 0\end{array}\right)$ & $\begin{array}{l}\text { Proper rotation through } \\
120^{\circ} \text { about } C_{3}(4) \text { axisd* } \\
\text { (fig.1). }\end{array}$ & (81276345) & $\left(\begin{array}{ccc}0 & 1 & 0 \\
0 & 0 & 1 \\
-1 & 0 & 0\end{array}\right)$ & $\begin{array}{l}\text { Inversion after the symmetry } \\
\text { element (or operation) } \mathrm{d} \text { (fig. } 1 \text { ). }\end{array}$ \\
\hline $\mathrm{f}$ & (18723654) & $\left(\begin{array}{lll}0 & 1 & 0 \\
0 & 0 & 1 \\
1 & 0 & 0\end{array}\right)$ & $\begin{array}{l}\text { Proper rotation through } \\
120^{\circ} \text { about } C_{3}(5) \text { axisf } \\
\text { (fig.1). }\end{array}$ & (63458127) & $\left(\begin{array}{ccc}0 & -1 & 0 \\
0 & 0 & -1 \\
-1 & 0 & 0\end{array}\right)$ & $\begin{array}{l}\text { Inversion after the symmetry } \\
\text { element (or operation) } \mathrm{f} \text { (fig.1). }\end{array}$ \\
\hline $\mathrm{g}$ & (32765814) & $\left(\begin{array}{ccc}0 & 0 & -1 \\
-1 & 0 & 0 \\
0 & 1 & 0\end{array}\right)$ & $\begin{array}{l}\text { Proper rotation through } \\
120^{\circ} \text { about } C_{3} \text { (6) axisg } \\
\text { (fig. } 1 \text { ). }\end{array}$ & (85412367) & $\left(\begin{array}{ccc}0 & 0 & 1 \\
1 & 0 & 0 \\
0 & -1 & 0\end{array}\right)$ & $\begin{array}{l}\text { Inversion after the symmetry } \\
\text { element (or operation) } g \text { (fig.1). }\end{array}$ \\
\hline $\mathrm{h}$ & (54367218) & $\left(\begin{array}{ccc}0 & 1 & 0 \\
0 & 0 & -1 \\
-1 & 0 & 0\end{array}\right)$ & $\begin{array}{l}\text { The proper rotation } \\
\text { through } 120^{\circ} \text { about } \mathrm{C}_{3} \mathrm{~h}^{*} \\
\text { (7) axis (fig.1). }\end{array}$ & (27814563) & $\left.\begin{array}{cc}-1 & 0 \\
0 & 1 \\
0 & 0\end{array}\right)$ & $\begin{array}{l}\text { Inversion after the symmetry } \\
\text { element (or operation) h (fig.1). }\end{array}$ \\
\hline i & (58143276) & 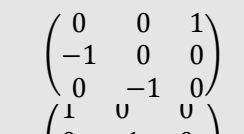 & $\begin{array}{l}\text { Proper rotation through } \\
120^{\circ} \text { about } C_{3}(8) \text { axisi }^{*} \\
\text { (fig. } 1 \text { ). } \\
\text { Proper rotation through }\end{array}$ & (23678541) & $\left(\begin{array}{ccc}0 & 0 & -1 \\
1 & 0 & 0 \\
0 & 1 & 0\end{array}\right)$ & $\begin{array}{l}\text { Inversion after the symmetry } \\
\text { element (or operation) i (fig.1). }\end{array}$ \\
\hline 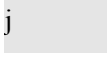 & (56781234) & $\left(\begin{array}{ccc}0 & -1 & 0 \\
n & n & -1\end{array}\right)$ & $\begin{array}{l}\text { Proper rotation through } \mathrm{j}^{*} \\
180^{\circ} \text { about } \mathrm{C}_{2}(1)(\mathrm{x})^{\mathrm{j}}\end{array}$ & (43218765) & $\left(\begin{array}{ccc}0 & -1 & 0 \\
n & n & 1\end{array}\right)$ & $\begin{array}{l}\text { Inversion after the symmetr } \\
\text { element (or operation) } \mathrm{k} \text { or refle }\end{array}$ \\
\hline
\end{tabular}


axis (fig.1).

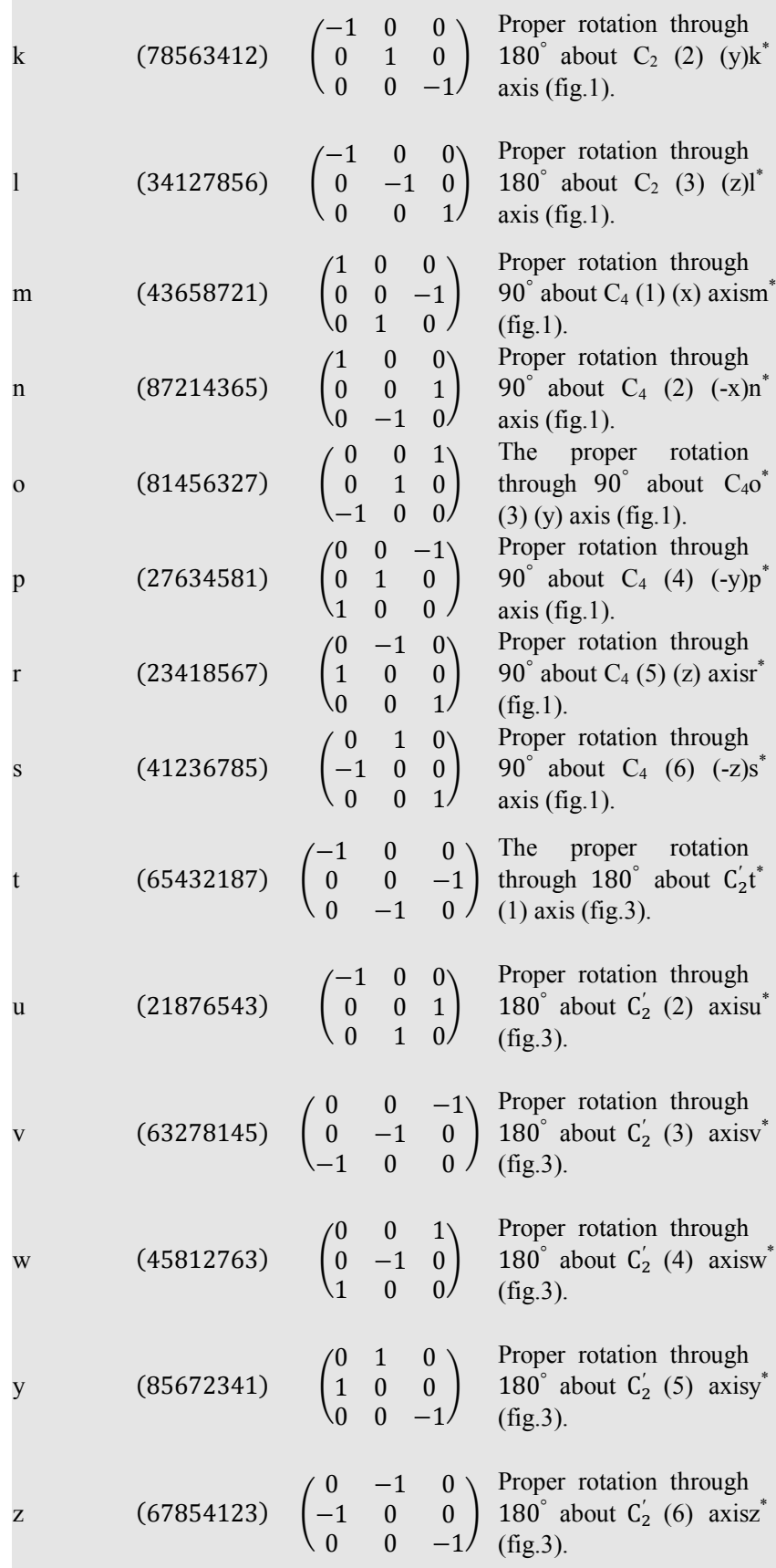

So far we have totally defined twenty four symmetry elements. Next one is the inversion, I, through the origin (or the centre of cube). The inversion converts the vector $r$ to the vector $-r$ and its permutation counterpart in $\mathrm{P}(8)$ is the element of (65872143). Each inversion performed after implementing the elements $\mathrm{a}, \mathrm{b}, \mathrm{c}, \mathrm{d}, \mathrm{f}, \mathrm{g}, \mathrm{h}$, and i, generates new symmetry elements which are $a^{*}, b^{*}, c^{*}, d^{*}, f^{*}$, $\mathrm{g}^{*}, \mathrm{~h}^{*}$, and $\mathrm{i}^{*}$ in Table 1 , respectively. For example element $\mathrm{a}^{*}$ is the inversion after the symmetry operation (or element) a, and its permutation counterpart in $\mathrm{P}(8)$ is the element of (67234185). Fig. 4 represents three reflection planes, ohs. Next symmetry element is the reflection through the plane $\sigma \mathrm{h}(1)$ in Fig. 4 and the symbol of this element is $j^{*}$, and its tion through the plane $\sigma_{\mathrm{h}}(1)$ (fig.4).

Inversion after the symmetry element (or operation) 1 or reflection through the plane $\sigma_{\mathrm{h}}(2)$ (fig.4)

Inversion after the symmetry element (or operation) $\mathrm{j}$ or reflection through the plane $\sigma_{\mathrm{h}}(3)$ (fig.4).

(78123456) $\quad\left(\begin{array}{ccc}-1 & 0 & 0 \\ 0 & 0 & 1\end{array}\right)$ Inversion after the symmetry element (or operation) m (fig.1).

(34567812) $\quad\left(\begin{array}{ccc}-1 & 0 & 0 \\ 0 & 0 & -1 \\ 0 & 1 & 0\end{array}\right) \begin{aligned} & \text { Inversion after the symmetry } \\ & \text { element (or operation) } n \text { (fig } 1 \text { ). }\end{aligned}$

(36721854) $\quad\left(\begin{array}{ccc}0 & 0 & -1 \\ 0 & -1 & 0 \\ 1 & 0\end{array}\right)$ Inversion after the symmetry element (or operation) o (fig.1). (54187236) $\quad\left(\begin{array}{ccc}0 & 0 & 1 \\ 0 & -1 & 0 \\ -1 & 0 & 0\end{array}\right) \begin{aligned} & \text { Inversion after the symmetry } \\ & \text { element (or operation) } \mathrm{p} \text { (fig.1). }\end{aligned}$

(58763214) $\quad\left(\begin{array}{ccc}0 & 1 & 0 \\ -1 & 0 & 0 \\ 0 & 0 & -1\end{array}\right) \begin{aligned} & \text { Inversion after the symmetry } \\ & \text { element (or operation) } r \text { (fig.1). }\end{aligned}$ (76581432) $\quad\left(\begin{array}{ccc}0 & -1 & 0 \\ 1 & 0 & 0 \\ 0 & 0 & -1\end{array}\right) \begin{aligned} & \text { Inversion after the symmetry } \\ & \text { element (or operation) s (fig.1.) }\end{aligned}$

(1 00 Inversion after the symmetry (56341278) $\quad\left(\begin{array}{ccc}1 & 0 & 0 \\ 0 & 0 & -1 \\ 0 & -1 & 0\end{array}\right) \begin{aligned} & \text { element (or operation) } \mathrm{u} \text { or reflec- } \\ & \text { tion through the plane } \sigma_{\mathrm{d}}(3487)\end{aligned}$ (fig.5).

(nversion after the symmetry element (or operation) t or reflection through the plane $\sigma_{\mathrm{d}}(1256)$ (fig.5). (72365418) $\quad\left(\begin{array}{ccc}0 & 0 & -1 \\ 0 & 1 & 0\end{array}\right) \begin{aligned} & \text { Inversion after the symmetry } \\ & \text { element (or operation) } w \text { or } \\ & \text { reflection through the plane } \sigma_{d}\end{aligned}$ reflection through the plane $\sigma_{d}$ (2358) (fig.5).

Inversion after the symmetry element (or operation) $\mathrm{v}$ or reflection through the plane $\sigma_{\mathrm{d}}(1467)$ (fig.5).

(32145876) $\quad\left(\begin{array}{ccc}0 & -1 & 0 \\ -1 & 0 & 0 \\ 0 & 0\end{array}\right) \begin{aligned} & \text { Inversion after the symmetry } \\ & \text { element (or operation) y or reflec- }\end{aligned}$ tion through the plane $\sigma_{\mathrm{d}}(2457)$ (fig.5).

Inversion after the symmetry element (or operation) z or reflection through the plane $\sigma_{\mathrm{d}}(1368)$ (fig.5). permutation counterpart is (43218765) in Table 1. This symmetry operation is actually equivalent to the symmetry operation which is the inversion after the symmetry operation k. Similarly, the symmetry elements $\mathrm{k}^{*}$ and $\mathrm{l}^{*}$ in Table 1 are the reflections through the planes $\sigma \mathrm{h}(2)$ and $\sigma \mathrm{h}(3)$ in Fig. 4, respectively, and they are equivalent to the inversions after the symmetry operations 1 and $\mathrm{j}$, respectively. The elements $\mathrm{m}^{*}, \mathrm{n}^{*}, \mathrm{o}^{*}, \mathrm{p}^{*}, \mathrm{r}^{*}$, and $\mathrm{s}^{*}$ in Table 1 represent the inversions after the symmetry operations $\mathrm{m}, \mathrm{n}, \mathrm{o}, \mathrm{p}, \mathrm{r}$, and s, respectively. Fig. 5 shows different reflection planes, бds. The symmetry operation which is the reflection through the plane $\sigma d$ (3487) in Fig. 5 or the inversion after symmetry operation $u$ is represented with symbol $t *$ in 
Table 1. While symmetry element $\mathrm{u}^{*}$ is the reflection through the plane $\sigma d(1256)$ in Fig. 5 or the inversion after symmetry operation $\mathrm{t}$, symmetry element $\mathrm{v}^{*}$ is the reflection through the plane $\sigma \mathrm{d}$ (2358) in Fig. 5 or the inversion after symmetry operation $\mathrm{w}$. Symmetry elements $\mathrm{w}^{*}$ and $\mathrm{y}^{*}$ are the reflections through the planes $\sigma d(1467)$ and $\sigma \mathrm{d}$ (2457) in Fig. 5, respectively, or the inversions after symmetry operations $\mathrm{v}$ and $\mathrm{y}$, respectively. And finally, the reflection through the plane $\sigma d$ (1368) in Fig. 5 or inversion after symmetry operation $\mathrm{z}$ is represented with symbol $\mathrm{z}^{*}$ in Table 1. Therefore we have totally defined all symmetry operations (or symmetry elements) of point group Oh, which leave the cube unchanged.

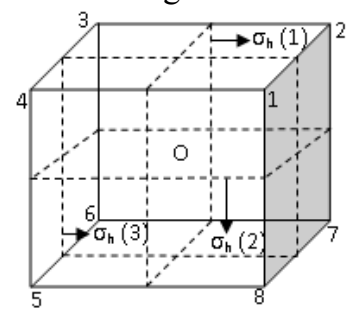

Figure 4. Illustration of reflection planes, $\sigma_{h}$, perpendicular to a principal axis of symmetry.

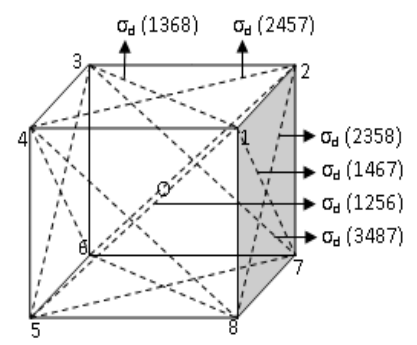

Figure 5. Illustration of reflection planes, $\sigma_{d}$, contain a principal axis of symmetry and bisect the angle between two two-fold axes perpendicular to the principal axis.

\section{Matrix Representations}

Seeing geometrically the results of successive applications of the symmetry operations is pretty hard. To overcome this difficulty we can find a matrix representation for every symmetry elements. The matrix representations enable us to perform the complicated and successive symmetry operations easily. The matrices form a group as well and they obey the same law of multiplication of the symmetry group itself. To explain finding the matrix which is corresponds to a symmetry element in the group, for example, let us find the matrix which is the representation of element $a$ in Table 1 using Fig. 6. The coordinates of corners of the cube are given in this figure. The corners of cube replace after symmetry operation $a$, as follows: $1 \rightarrow 1,2 \rightarrow 4,3 \rightarrow 5$, $4 \rightarrow 8,5 \rightarrow 7,6 \rightarrow 6,7 \rightarrow 3$, and $8 \rightarrow 2$. The matrix A which creates this change in the coordinates of corners should be in the form

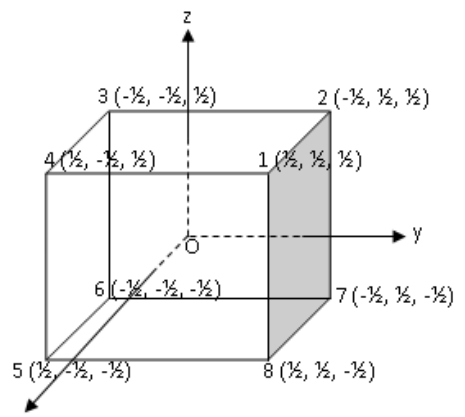

Figure 6. Illustration of the coordinates of corners of cube.

$$
A=\left(\begin{array}{lll}
a_{11} & a_{12} & a_{13} \\
a_{21} & a_{22} & a_{23} \\
a_{31} & a_{32} & a_{33}
\end{array}\right)
$$

To determine the coefficients $a_{i j}$ we need nine equations which contain the coefficients $a_{i j}$ themselves. The systems of equation $A r_{1}=r_{1}, A r_{2}=r_{4}, A r_{3}=r_{5}$ give the equations we need; where $\mathrm{A}$ is the matrix corresponds to the symmetry element a, $r_{1}, r_{2}, r_{3}, r_{4}$, and $r_{5}$ are the coordinates of the corners $1,2,3,4$, and 5 , respectively. Therefore we obtain the equations,

$$
\begin{aligned}
& a_{11}+a_{12}+a_{13}=1, a_{21}+a_{22}+a_{23}=1, a_{31}+a_{32}+ \\
& a_{33}=1 \\
& -a_{11}+a_{12}+a_{13}=1,-a_{21}+a_{22}+a_{23}=-1,-a_{31}+ \\
& a_{32}+a_{33}=1 \\
& -a_{11}-a_{12}+a_{13}=1,-a_{21}-a_{22}+a_{23}=-1,-a_{31}- \\
& a_{32}+a_{33}=-1
\end{aligned}
$$

from the systems of equation above. Solving these equations together we find the coefficients $a_{i j}$, therefore, the A becomes

$$
A=\left(\begin{array}{lll}
0 & 0 & 1 \\
1 & 0 & 0 \\
0 & 1 & 0
\end{array}\right)
$$

We can similarly find the matrix representations of all symmetry elements which are given in Table 1.

The multiplication table of symmetry group $\mathrm{O}_{\mathrm{h}}$, obtained using the permutation counterparts or matrix representations of all symmetry elements of symmetry group $\mathrm{O}_{\mathrm{h}}$, is given in Table 2. To obtain multiplication of any two elements in the group one can use the permutation counterparts of the elements, for example,

$$
a b=\left(\begin{array}{l}
12345678 \\
14587632
\end{array}\right)\left(\begin{array}{l}
12345678 \\
72185436
\end{array}\right)=\left(\begin{array}{l}
12345678 \\
34127856
\end{array}\right)=l,
$$

or use the matrix representations of them,

$A B=\left(\begin{array}{lll}0 & 0 & 1 \\ 1 & 0 & 0 \\ 0 & 1 & 0\end{array}\right)\left(\begin{array}{ccc}0 & -1 & 0 \\ 0 & 0 & 1 \\ -1 & 0 & 0\end{array}\right)=\left(\begin{array}{ccc}-1 & 0 & 0 \\ 0 & -1 & 0 \\ 0 & 0 & 1\end{array}\right)=l$

Using Table 2 one easily can prove that the set of forty eight elements provide the group axioms. Every row or 
column in the multiplication table in Table 2 contains every element once. Hence, the first group axiom, the closure, is provided. The second axiom, the existence of unit element is already provided. Third axiom, the existence of inverse of every element, can be seen in the multiplication table. If we go to the right along the row of any element in the mul- tiplication table, we really reach the unit element. If we go up from here we can reach the inverse element for element under consideration. Using the multiplication table, it can be easily shown that the fourth axiom, the associative law, is provided.

Table 2. Multiplication table of point group $O_{h}$.

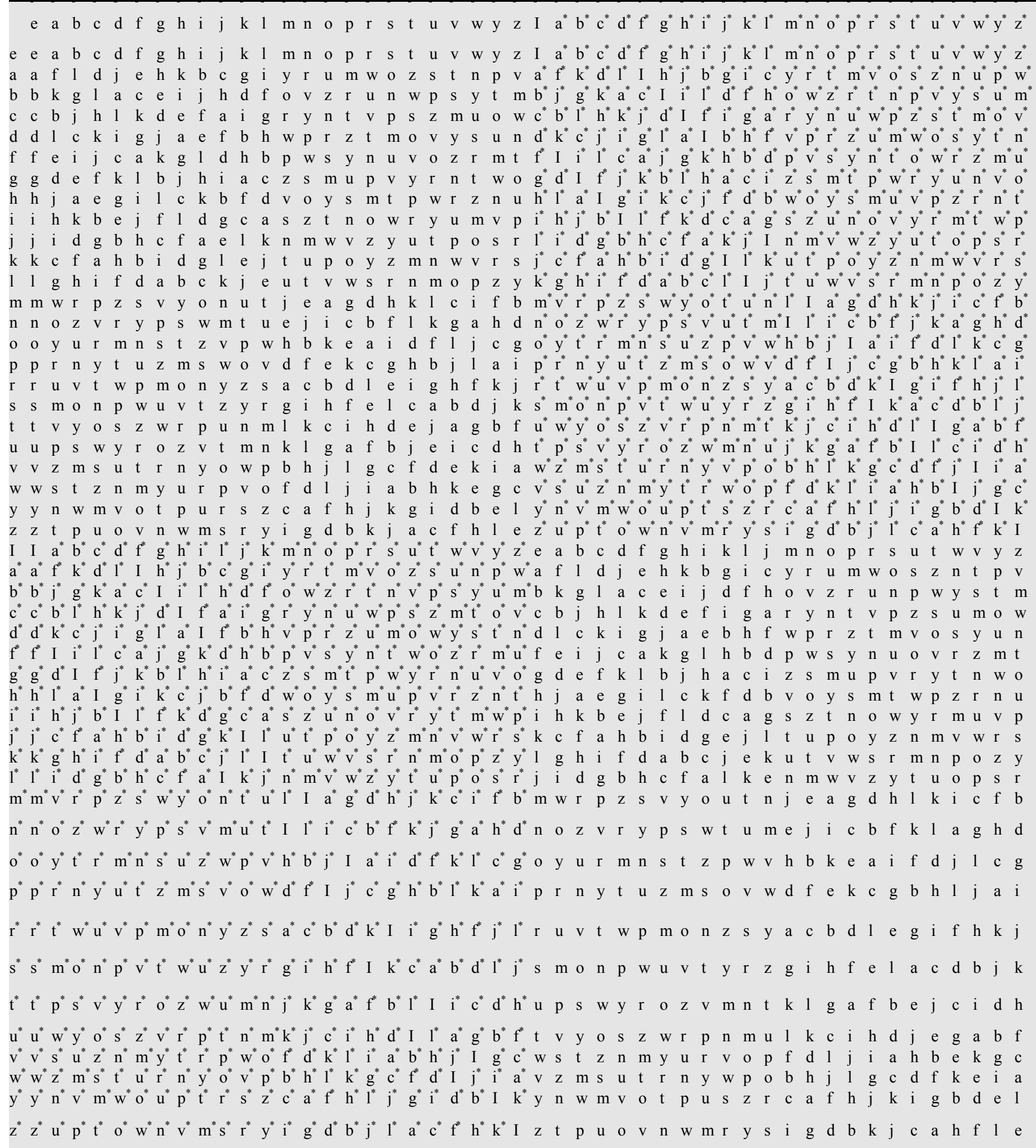

\section{Classes}

Now let us determine the orders of elements and all classes in the group. The order of any element $a$ in a given 
group is determined using the relation

$$
a^{n}=e .
$$

The smallest value of $\mathrm{n}$ in the relation above is the order of element a. For example, for element $a^{*}$ in Table 1 we can show using Table 2 that

$$
\begin{gathered}
a^{* 6}=a^{* 4} a^{*} a^{*}=a^{* 4} f=a^{* 3} a^{*} f=a^{* 3} I=a^{* 2} a^{*} I= \\
a^{* 2} a=a^{*} a^{*} a=a^{*} f^{*}=e .
\end{gathered}
$$

Then, the order of element $a^{*}$ becomes six. The obtained orders of elements in symmetry group $\mathrm{O}_{\mathrm{h}}$ using the relation above are given in Table 3.

Table 3. Orders of all elements in point group $O_{h}$.

\begin{tabular}{ll}
\hline Order & Symmetry elements \\
\hline 1 & e \\
2 & $j, k, l^{*}, \mathrm{k}^{*}, \mathrm{l}^{*}, \mathrm{t}, \mathrm{u}, \mathrm{v}, \mathrm{w}, \mathrm{y}, \mathrm{z}, \mathrm{I}_{\mathrm{t}} \mathrm{t}^{*}, \mathrm{u}^{*}, \mathrm{v}^{*}, \mathrm{w}^{*}, \mathrm{y}^{*}, \mathrm{z}^{*}$ \\
3 & $\mathrm{a}, \mathrm{b}, \mathrm{c}, \mathrm{d}, \mathrm{f}, \mathrm{g}, \mathrm{h}, \mathrm{i}$ \\
4 & $\mathrm{~m}, \mathrm{n}, \mathrm{o}, \mathrm{p}, \mathrm{r}, \mathrm{s}, \mathrm{m}^{*}, \mathrm{n}^{*}, \mathrm{o}^{*}, \mathrm{p}^{*}, \mathrm{r}^{*}, \mathrm{~s}^{*}$ \\
6 & $\mathrm{a}^{*}, \mathrm{~b}^{*}, \mathrm{c}^{*}, \mathrm{~d}^{*}, \mathrm{f}^{*}, \mathrm{~g}^{*}, \mathrm{~h}^{*}, \mathrm{i}^{*}$ \\
\hline
\end{tabular}

To find the classes in a given group we use the conjugation. If $a, b$, and $c$ are three arbitrary elements in the group, and if the elements $a$ and $b$ satisfy the condition

$$
a=c b c^{-1}
$$

then the element $a$ conjugate to the element $b$, or vice versa, since

$$
b=c^{-1} a c
$$

the element $b$ conjugate to the element $a$ as well. An arbitrary element in a given group and all elements which obtained from this element by conjugation form a class for the group. All elements which belong to same class have the same order. Thus Table 3 can give a prior idea about the answer of question that which elements can be form same class? For example, let us use the relation of conjugation for the element $j$. If we use the element $j$ and all elements in group $\mathrm{O}_{\mathrm{h}}$ instead of $b$ and $c$ in the relation, respectively,

$$
\begin{gathered}
e j e^{-1}=j j j^{-1}=k j k^{-1}=l j l^{-1}=m j m^{-1}=n j n^{-1}= \\
t j t^{-1}=u j u^{-1}=I j I^{-1}=j^{*} j j^{*-1}=k^{*} j k^{*-1}=l^{*} j l^{*-1}= \\
m^{*} j m^{*-1}=n^{*} j n^{*-1}=t^{*} j t^{*-1}=u^{*} j u^{*-1}=j, \\
a j a^{-1}=c j c^{-1}=g j g^{-1}=i j i^{-1}=r j r^{-1}=s j s^{-1}= \\
y j y^{-1}=z j z^{-1}=a^{*} j a^{*-1}=c^{*} j c^{*-1}=g^{*} j g^{*-1}= \\
i^{*} j i^{*-1}=r^{*} j r^{*-1}=s^{*} j s^{*-1}=y^{*} j y^{*-1}=z^{*} j z^{*-1}=k, \\
b j b^{-1}=d j d^{-1}=f j f^{-1}=h j h^{-1}=o j o^{-1}=p j p^{-1}= \\
v j v^{-1}=w j w^{-1}=b^{*} j b^{*-1}=d^{*} j d^{*-1}=f^{*} j f^{*-1}= \\
h^{*} j h^{*-1}=o^{*} j o^{*-1}=p^{*} j p^{*-1}=v^{*} j v^{*-1}=w^{*} j w^{*-1}=l,
\end{gathered}
$$

then we get the elements $j, k$, and $l$. Thus we see that the elements $j, k$, and $l$ form a class. The obtained all other classes for the symmetry group $\mathrm{O}_{\mathrm{h}}$, using the definitions of conjugation and class, are presented in Table 4.

Table 4. All classes in point group $O_{h}$.

\begin{tabular}{ll}
\hline & Symmetry elements \\
\hline Class 1: $\mathrm{e}$ & $\mathrm{e}, \mathrm{k}, \mathrm{l}$ \\
Class 2: $3 \mathrm{C}_{2}$ & $\mathrm{j}^{*}, \mathrm{k}^{*}, \mathrm{l}^{*}$ \\
Class 3: $3 \sigma_{\mathrm{h}}$ & $\mathrm{t}, \mathrm{u}, \mathrm{v}, \mathrm{w}, \mathrm{y}, \mathrm{z}$ \\
Class 4: $6 \mathrm{C}_{2}^{\prime}$ & $\mathrm{I}$ \\
Class 5: $\mathrm{I}$ & $\mathrm{t}^{*}, \mathrm{u}^{*}, \mathrm{v}^{*}, \mathrm{w}^{*}, \mathrm{y}^{*}, \mathrm{z}^{*}$ \\
Class 6: $6 \sigma_{\mathrm{d}}$ & $\mathrm{a}, \mathrm{b}, \mathrm{c}, \mathrm{d}, \mathrm{f}, \mathrm{g}, \mathrm{h}, \mathrm{i}$ \\
Class 7: $8 \mathrm{C}_{3}$ & $\mathrm{~m}, \mathrm{n}, \mathrm{o}, \mathrm{p}, \mathrm{r}, \mathrm{s}$ \\
Class 8: $6 \mathrm{C}_{4}$ & $\mathrm{~m}^{*}, \mathrm{n}^{*}, \mathrm{o}^{*}, \mathrm{p}^{*}, \mathrm{r}^{*}, \mathrm{~s}^{*}$ \\
Class 9: $6 \mathrm{~S}_{4}$ & $\mathrm{a}^{*}, \mathrm{~b}^{*}, \mathrm{c}^{*}, \mathrm{~d}^{*}, \mathrm{f}^{*}, \mathrm{~g}^{*}, \mathrm{~h}^{*}, \mathrm{i}^{*}$ \\
\hline
\end{tabular}

\section{Subgroups}

Let a be any element in a given group. The set elements $e, a, a^{2}, a^{3}, a^{n-1}$ is called the period of element $\mathrm{a}$, where $\mathrm{n}$ is the order of a. The period forms an Abelian subgroup. Let us now find the periods of elements in group Oh. For example, the order of element $a^{*}$ is six, therefore, the period of $a^{*}$ becomes

$$
\left\{e, a^{*}, a^{* 2}, a^{* 3}, a^{* 4}, a^{* 5}\right\}=\left\{e, I, a, a^{*}, f, f^{*}\right\}
$$

which is an Abelian subgroup of group $\mathrm{O}_{\mathrm{h}}$. The obtained all Abelian subgroups for group $\mathrm{O}_{\mathrm{h}}$, using the definition of the period of an element, are given below:

$\{e\},\{e, j\},\{e, k\},\{e, l\},\{e, t\},\{e, u\},\{e, v\},\{e, w\},\{e, y\}$, $\{e, z\},\{e, I\},\left\{e, j^{*}\right\},\left\{e, k^{*}\right\},\left\{e, l^{*}\right\},\left\{e, t^{*}\right\},\left\{e, u^{*}\right\},\left\{e, v^{*}\right\}$, $\left\{e, w^{*}\right\},\left\{e, y^{*}\right\},\left\{e, z^{*}\right\},\{e, a, f\},\{e, b, g\},\{e, c, h\},\{e, d, i\}$, $\{e, j, m, n\},\{e, k, o, p\},\{e, l, r, s\},\left\{e, j, m^{*}, n^{*}\right\},\left\{e, k, o^{*}, p^{*}\right\}$, $\left\{e, l, r^{*}, s^{*}\right\},\left\{e, I, a, a^{*}, f, f^{*}\right\},\left\{e, I, b, b^{*}, g, g^{*}\right\}$ $\left\{e, I, c, c^{*}, h, h^{*}\right\},\left\{e, I, d, d^{*}, i, i^{*}\right\}$.

In group theory, there is no any theorem which can give us the number of subgroups for a given finite group. Even so, we can construct some subgroups according to one (or more) element (or elements). For example, let us construct a subgroup according to elements e, $a$, and $b$ in Table 1 . We have now a set of elements: $\{e, a, b\}$. However, we see that this set is not a subgroup because it does not contain the inverse elements of $a$ and $b$. So, we should add the elements $\mathrm{f}$ and $\mathrm{g}$, the inverses of $\mathrm{a}$ and $\mathrm{b}$, to this set. We have $\mathrm{a}$ new set $\{e, a, b, f, g\}$. This new set cannot be a subgroup as well because its order is not a divisor of the order of group $\mathrm{Oh}$ (the order of a group is its number of elements and the order of a subgroup has to be a divisor of the order of the group). Furthermore, it does not satisfy the closure axiom. Therefore, we see that it has to include the elements c, d, h, 
i, k, l. Namely, the set should be $\{e, a, b, c, d, f, g, h, i, k, l\}$. But the set is not a subgroup yet because it is not close with respect to the law of multiplication. The order of this set is also not a divisor of the order of group Oh. We easily see that the set must contain element $j$ as well in order to satisfy the first group axiom, the closure. The set of $\{e, a, b, c, d, f, g, h, i, j, k, l\}$ is now a subgroup of group Oh. It can easily be shown that the set satisfies four group axioms, that is, it is a subgroup of group Oh. Some subgroups of group $\mathrm{Oh}$, which are obtained using the same method above according to the set of elements $\left\{e, a^{*}, z^{*}\right\}$, $\left\{e, b^{*}, y^{*}\right\},\left\{e, c^{*}, v^{*}\right\},\left\{e, d^{*}, w^{*}\right\},\{e, a, m\},\left\{e, a, a^{*}\right\}$, and $\left\{e, a, m^{*}\right\}$, respectively, are given below:

$$
\begin{aligned}
& \left\{e, a, f, t, v, z, I, a^{*}, f^{*}, u^{*}, w^{*}, z^{*}\right\}, \\
& \left\{e, b, g, t, w, y, I, b^{*}, g^{*}, u^{*}, v^{*}, y^{*}\right\}, \\
& \left\{e, c, h, u, w, z, I, c^{*}, h^{*}, t^{*}, v^{*}, z^{*}\right\}, \\
& \left\{e, d, i, u, v, y, I, d^{*}, i^{*}, t^{*}, w^{*}, y^{*}\right\},
\end{aligned}
$$

$\{e, a, b, c, d, f, g, h, i, j, k, l, m, n, o, p, r, s, t, u, v, w, y, z\}$, $e, a, b, c, d, f, g, h, i, j, k, l, m *, n *, o *, r *, s *, t *, u *, \imath *, w *$, $y^{*}, z^{*}, e, a, b, c, d, f, g, h, i, j, k, l, l, a *, b *, c *, d *, f *, g^{*}$, $h *, i *, j *, k *, l *$.

The subgroups of

$\{e, a, b, c, d, f, g, h, i, j, k, l, m, n, o, p, r, s, t, u, v, w, y, z\}$

and

$\left\{e, a, b, c, d, f, g, h, i, j, k, l, I, a^{*}, b^{*}, c^{*}, d^{*}, f^{*}, g^{*}, h^{*}, i^{*}, j^{*}, k^{*}, l^{*}\right.$ correspond to the point groups $\mathrm{O}$ and $\mathrm{T}_{h}$, respectively.

\section{Conclusions}

We have clearly defined all elements of point group $\mathrm{O}_{\mathrm{h}}$ using appropriate figures. Then we have found the matrix representations for every element. We have formed the multiplication table for point group $\mathrm{O}_{\mathrm{h}}$ using the matrix representations and the permutation counterparts for every element. Furthermore, we have determined the order and period of every element, then, we have found all classes. Finally, we have found all Abelian subgroups and some other subgroups of $\mathrm{O}_{h}$.

\section{References}

[1] H. F. Jones, "Group, Representations and Physics", London: IOP Publishing, 1998.

[2] L. Mariot, "Group Theory and Solid State Physics", London: Prentice-Hall International Inc, 1962.

[3] W. Ledermann, "Introduction to Group Theory", London: Longman, 1973.

[4] U. Shmueli, "Theories and Techniques of Crystal Structure Determination”, Oxford: Oxford University Press, 2007.

[5] P. Ramond, "Group Theory A Physicist's Survey", Cambridge: Cambridge University Press, 2010.

[6] A. O. Barut and R. Raczka, "Theory of Group Representations and Applications", Warszawa: Polish Scientific Publishers, 1980.

[7] Z. -Q. Ma and X. -Y. Gu, "Problems and solutions in Group Theory for Physicists", Singapore: World Scientific Publishing, 2004.

[8] J. Q. Chen, J. Ping and F. Wang, "Group Representation Theory for Physicists", Singapore: World Scientific Publishing, 2002.

[9] M. S. Dresselhaus, G. Dresselhaus and A. Jorio, "Group Theory Application to the Physics of Condensed Matter", Berlin-Heidelberg: Springer-Verlag, 2008.

*Y10] M. A. Armstrong, "Groups and Symmetry", BerlinHeidelberg: Springer-Verlag, 1988.

[11] M. Hamermesh, "Group Theory and Its Applications to Physical Problems”, New York: Dover, 1989.

[12] F. D. Murnaghan, "The Theory of Group Representations", Baltimore: The Johns Hopkins Press, 1938.

[13] M. A. Naimark and A. I. Stern, "Theory of Group Representations", New York: Springer-Verlag, 1982.

[14] G. F. Koster, "Space Groups and Their Representations" New York and London: Academic Press, 1957.

[15] N. W. Ashcroft and N. D. Mermin, "Solid State Physics" New York: Saunders College Publishing, 1976. 